\title{
Vom Arbeitgeber zur Marke
}

\section{"Employer Branding" als Hilfe zur Organisationsentwicklung und Personalgewinnung}

\author{
CHRISTOPHER ROBBERG \\ Christopher Roßberg ist Organisa- \\ tionsberater bei der Unternehmens- \\ beratung contec $\mathrm{GmbH}$ in der \\ Geschäftsstelle Stuttgart. Seine \\ Tätigkeitsschwerpunkte liegen im \\ Personalmarketing und Employer \\ Branding für Träger der Alten- und \\ Behindertenhilfe. \\ rossberg@contec.de
}

\author{
Organisationen und Unternehmen, die als glaubwürdige \\ und attraktive Arbeitgeber gelten, haben es \\ selbstverständlich leichter, Mitarbeitende zu halten \\ und neue zu gewinnen. Die Entwicklung einer eigenen \\ "Arbeitgebermarke» kann diesen Prozess unterstützen.
}

Symptome: lange Vakanzen, unpassende Bewerber, fehlender Nachwuchs, hohe Personalmarketingkosten, Überlastung des Personals und steigende Fluktuation. Diagnose: Fachkräftemangel. In den verschiedensten Bereichen der Sozialwirtschaft, von der Kita bis zum Pflegeheim, lässt sich heute diese »Krankheit« attestieren.

Ausreichend qualifiziertes Personal ist zur Mangelerscheinung geworden. Doch welches Rezept hilft? Kurzfristig mehr Geld in Stellenanzeigen investieren, »Kopfgeldprämien « für das Werben von neuen Mitarbeitern ausgeben oder einfach nur abwarten?

Eine Strategie, um als soziale Einrichtung langfristig resistent gegen den Fachkräftemangel zu sein, ist die
Entwicklung einer »Arbeitgebermarke«. Die Entwicklung, Positionierung und Steuerung dieser Arbeitgebermarke wird als »Employer Branding « bezeichnet. In diesem Artikel wird zunächst auf das Endprodukt, die Arbeitgebermarke mit ihren Wirkungen und Funktionen, eingegangen und im Anschluss der Employer-BrandingProzess, also der Weg zur Arbeitgebermarke beschrieben. Im Vorfeld ist festzuhalten, dass sich das Konzept des Employer Branding unabhängig von der Größe des Unternehmens anwenden lässt. Vom einzelnen Pflegeheim über einen KITA-Verbund bis hin zum großen Komplexträger können sich Einrichtungen zur Arbeitgebermarke entwickeln.

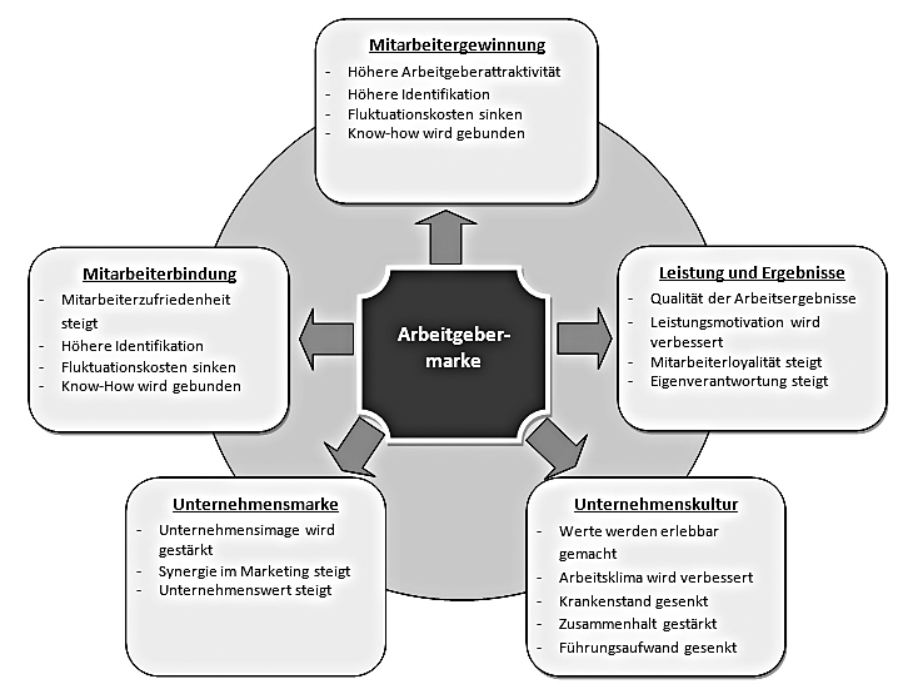

Die Deutsche Employer Branding Akademie benennt fünf Wirkungskreise einer "Arbeitgebermarke". 


\section{Die Arbeitgebermarke}

Die wissenschaftlich gängigste Definition bezeichnet Employer Branding "als eine identitätsbasierte, intern wie extern wirksame Entwicklung und Positionierung eines Unternehmens als glaubwürdiger und attraktiver Arbeitgeber« (Kriegler, 2012). Ein Unternehmen ist demnach zur Arbeitgebermarke geworden, wenn die relevanten Bezugsgruppen ein überwiegend einheitliches und unterscheidbares Vorstellungsbild von ihm als Arbeitgeber haben. Eine Arbeitgebermarke wirkt nach innen und nach außen. Die Deutsche Employer Branding Akademie ermittelte hierfür empirisch fünf Wirkungskreise einer Arbeitgebermarke (vgl. Abb.).

Die Wirkungskreise zeigen, dass es sich beim Employer Branding um Markenbildung im Sinne einer Identitätsund Organisationsentwicklung handelt, welche weit über das externe Personalmarketing hinausgeht. Der Weg zu einer Arbeitgebermarke lässt sich in fünf Phasen einteilen:

\section{- Vorbereitung und Set-up}

- Analyse

- Entwicklung der Arbeitgeberpositionierung

- interne und externe Implementierung

- Steuerung

Je nach Größe des Unternehmens und Fortschritt im Prozess dauert die Etablierung als Arbeitgebermarke zwei bis fünf Jahre.

\section{Vorbereitung und Set-up}

Employer Branding ist eine strategische Entscheidung, die auf der obersten Ebene des Vorstands und der Geschäftsführung gefällt wird. Auf dieser Ebene muss Klarheit über die Ziele herrschen und ein ge- meinsames Verständnis über die Bedeutung des Projektes gewonnen werden. Für die Projektorganisation werden eine Projektgruppe, ein Lenkungskreis und gegebenenfalls ein Soundboard eingesetzt. Die Größe und Besetzung variiert je nach der Größe und Struktur der Einrichtung oder des Trägers (vgl. Tabelle).

Der Start des Projektes findet in einer gemeinsamen Auftaktveranstaltung statt, bei der die Führungskräfte und Mitarbeiter über die Ziele und den Projektverlauf informiert werden. Es empfiehlt sich außerdem, ein Informationsschreiben an alle weiteren Mitarbeiter herauszugeben, die nicht an der Veranstaltung teilnehmen können.

\section{Analysephase}

Diese Phase im Employer Branding stellt die empirisch fundierte Grundlage für die Entwicklung der Arbeitgebermarke dar. Sie besteht aus den Bausteinen: Analyse der Zielgruppenpräferenzen, der Rahmenbedingungen, der Soll-Perspektive und der Unternehmenskultur.

Zunächst muss Klarheit über die Zielgruppe und deren Präferenzen geschaffen werden. Welche Berufsgruppe wollen wir ansprechen (z. B. Pflegefachkräfte, Erzieherinnen, Sozialpädagogen)? Jede Berufsgruppe hat verschiedene Präferenzen in Bezug auf ihre Wahl des Arbeitgebers und Wünsche, wie der Arbeitsplatz und der Arbeitsalltag aussehen sollen. Deswegen lohnt es sich oft, neben einer Recherche nach passenden Studien, eine Kurzumfrage mit verschieden Items (z. B. aus den Bereichen Werte, Vergütung, Entwicklung, Führung, Arbeitsplatzgestaltung) unter den eigenen Mitarbeitern durchzuführen. Diese können in der Regel sehr genau benennen, welche Präferenzen sie in Bezug auf den Arbeits- platz haben. In diesem Zuge kann auch ein Bild des Arbeitgeberimages erhoben werden. Die eigenen Mitarbeiter haben oft ein gutes Gespür dafür, wie ihr Arbeitgeber extern wahrgenommen wird.

Die Rahmenbedingungen zu kennen, in denen man sich als Arbeitgeber bewegt, ist entscheidend für die spätere Positionierung der Arbeitgebermarke. Hierfür findet eine Wettbewerbsanalyse mit folgenden Untersuchungsgegenständen statt: Arbeitgeberimage, Konkurrenz um gleiche Bewerber, Darstellung der Unternehmenskultur, Personalmarketingaktivitäten. Des Weiteren werden die regionalen Arbeitsmarktentwicklungen mit einbezogen. Um sich über die Entwicklungen der eigenen Belegschaft ein Bild zu machen, sind außerdem Personalkennzahlen wie Fluktuation, Bewerber pro Stelle, Altersentwicklung etc. hilfreich.

Für die Erhebung der Soll-Perspektive sollten Interviews mit der operativen Geschäftsführung über die Vision der Einrichtung, die Unternehmenskultur, die Kommunikation und Führung, den idealen Mitarbeitertypus und die strategische Planung der Zukunft geführt werden. Diese Soll-Perspektive gibt später eine Orientierung, wohin sich die Arbeitgebermarke entwickeln soll.

Die Kulturanalyse mit den Mitarbeitenden ist der wichtigste Bestandteil der Analysephase. Es ist eine Bestandsaufnahme all jener Merkmale, Faktoren und Haltungen, die das Erleben des Arbeitgebers seitens der Mitarbeiter und Führungskräfte prägen - positiv wie negativ. Es handelt sich um Kulturfaktoren, Identitätsmerkmale und Wertehaltungen des Unternehmens, die sich beim Arbeitnehmer unter anderem auf die Leistungsbereitschaft, das Zufriedenheitsgefühl und seine Wechselbereitschaft auswirken.

\begin{tabular}{|c|c|c|}
\hline & Besetzung & Aufgaben \\
\hline Projektteam & $\begin{array}{l}\text { Personalakquise(z. B. Pflegedienstleitung, Wohngruppenleitung, } \\
\text { Personalleitung) } \\
\text { Öffentlichkeitsarbeit, Marketing (z. B. Referent für } \\
\text { Öffentlichkeitsarbeit, Verwaltungsmitarbeiter) } \\
\text { Personal - und Organisationsentwicklung (z. B. Referent für } \\
\text { Personalentwicklung, Stabstellenmitarbeiter) }\end{array}$ & $\begin{array}{l}\text { Projektleitung } \\
\text { aktive Planung und Umsetzung } \\
\text { der Maßnahmen }\end{array}$ \\
\hline Lenkungskreis & $\begin{array}{l}\text { Geschäftsführung } \\
\text { Vorstand } \\
\text { Mitarbeitervertretung, Betriebsrat }\end{array}$ & $\begin{array}{l}\text { Richtungsentscheidungen } \\
\text { Unterstützung bei der } \\
\text { Akzeptanz }\end{array}$ \\
\hline Soundboarding & $\begin{array}{l}\text { Mitarbeiter aus verschiedenen Einrichtungen } \\
\text { (z. B. Wohnbereichsleitungen, Gruppenleiter) } \\
\text { Mitarbeitervertretung, Betriebsrat }\end{array}$ & $\begin{array}{l}\text { Denkanstöße, Impulse } \\
\text { - Feedback }\end{array}$ \\
\hline
\end{tabular}


Für diese Analyse werden Fokusgruppen gegründet, die acht bis zwölf Mitarbeitende groß sind und die, wenn möglich, mit einem externen Moderator an den Fragestellungen zur Unternehmenskultur arbeiten. Bei der Zusammenstellung der Gruppen ist es wichtig, Mitarbeitende von Führungskräften zu trennen, um eine offene Atmosphäre zu schaffen. Die Besetzung der Fokusgruppen kann nach Berufsbild, Alter, Betriebszugehörigkeit, Geschlecht etc. variieren und sollte an dem Ziel des Employer Branding ausgerichtet werden (z. B. mehr männliche Erzieher gewinnen, erfordert eine Fokusgruppe mit ausschließlich Männern).

Pflicht ist es, eine Fokusgruppe einzurichten mit ausschließlich neuen Mitarbeitenden, die nicht länger als ein ne Arbeitgeberpositionierung gibt Antwort auf folgende Leitfragen:

- Werte: Wofür stehe ich als Arbeitgeber? (Identifikationsfaktoren)

- Ziele: Wo will ich hin? (Identifikationsfaktoren)

- Identität: Was macht mich besonders? (Differentiatoren)

- Kultur: Wer passt zu mir? (Differentiatoren)

Die Arbeitgeberpositionierung hat eine Identifikationsfunktion für Mitarbeiter und Führungskräfte, eine Differenzierungsfunktion in den Arbeitsmärkten und eine Orientierungsfunktion für Bewerber. Um diese Funktionen zu erfüllen, muss eine Positionierung authentisch und glaub-

\section{"Die eigenen Mitarbeiter haben ein gutes Gespür, wie ihr Arbeitgeber extern wahrgenommen wird"}

Jahr im Unternehmen sind. Diese Gruppe hat oft noch ein unverfälschtes Bild und kann am besten einen Vergleich zu früheren Arbeitgebern ziehen. Die Gruppenanzahl lässt sich individuell, bezogen auf die Geschäftsfelder und die Größe der Einrichtung bestimmen. Für eine einheitliche Positionierung als Arbeitgeber ist wichtig, dass jeder Geschäftsbereich sich involviert fühlt, weil sonst eine allgemeine Akzeptanz der Ergebnisse nicht gewährleistet ist.

\section{Entwicklung der Arbeitgeberpositionierung}

Die Arbeitgeberpositionierung ist das Herzstück der Arbeitgebermarke und wird aus den Ergebnissen der Analysephase entwickelt. Hohe Relevanz haben die Soll-Perspektive der Geschäftsführung, weil sie die Leitplanken und Entwicklungen der Zukunft definiert, sowie die erhobenen Identitäts- und Kulturfaktoren und gelebten Werte aus der Kulturanalyse.

Die weiteren Ergebnisse aus der Analysephase gilt es hier ebenfalls zu berücksichtigen. Aus den Ergebnissen werden Profilthemen abgeleitet, validiert durch den Lenkungskreis und das Soundboard und im Anschluss zu einer Positionierung formuliert. Eine gelunge- würdig sein. Positionierungen, die an der Realität vorbei formuliert sind, werden schnell als falsch entlarvt und können entgegengesetzte Wirkungen entfalten.

\section{Interne und externe Implementierung}

Der nächste Schritt im Employer Branding ist nun, die entwickelte Positionierung intern und extern zu implementieren. Führungskräfte nehmen bei der internen Implementierung eine Schlüsselfunktion ein. Sie sind Botschafter im Sinne der Kommunikation, aber auch durch ihre an der formulierten Positionierung orientierte Führung und Haltung gegenüber den Mitarbeitern. Die Bekanntmachung gegenüber den Mitarbeitenden sollte zweigleisig erfolgen: einerseits mit klassischen Kommunikationsmedien wie Newsletter, Mitarbeiterzeitung und Intranet, andererseits im Dialog und Austausch. Für Letzteres sollte Raum und Zeit gegeben und nicht nur nebenbei, beispielsweise bei Übergaben oder Teamsitzungen, kurz gesprochen werden. Im besten Fall werden Mitarbeiter zu Markenbotschaftern und unterstützen die externe Implementierung der Arbeitgebermarke.

Für die externe Positionierung sind Kommunikationsmedien wie die Homepage, Flyer, Social Media, Stel- lenanzeigen wichtig. Ein ansprechender, aktueller Internetauftritt mit einer passenden Karriereseite bietet die ideale Plattform zur Kommunikation der erarbeiteten Positionierung. Beispielsweise mit Videos, Interviews mit Mitarbeitenden oder einer Bewerbersprechstunde können die Werte, Ziele, Identität und Kultur lebensnah dargestellt werden. Die Ergebnisse der Wettbewerbsanalyse in Bezug auf das Personalmarketing der anderen Wettbewerber am Arbeitsmarkt finden hier Anwendung und dienen als Grundlage für differenzierte, externe Personalmarketingaktivitäten.

\section{Steuerung}

Nach der internen und externen Implementierung der Arbeitgeberpositionierung kann die Entwicklung des Unternehmens zur Arbeitgebermarke mit Hilfe von Kennzahlen gesteuert werden. Als Instrumente dienen regelmäßige Mitarbeiterumfragen, beispielsweise mit Fragen zum Identifikationsgrad, der Intensität des Erlebens der formulierten Werte und Glaubwürdigkeit der Positionierung. Der Erfolg der Arbeitgebermarke lässt sich mit Erhebung von Personalkennzahlen messen, beispielsweise der Bewerberanzahl, Fluktuationsrate, dem Krankenstand. Bei Abweichungen sollte das Projektteam korrigierend eingreifen.

\section{Fazit}

Soziale Einrichtungen scheuen heutzutage noch aufgrund der langfristigen Dauer, der Kosten und der fehlenden Expertise den Weg zur Arbeitgebermarke zu gehen. Manche Regionen und Bereiche der Sozialwirtschaft sind auch schon so sehr vom Fachkräftemangel betroffen, dass kurzfristige Maßnahmen gesucht werden.

Employer Branding muss deshalb als strategische Entscheidung, als Investition in die Zukunft, verstanden werden. Ein Blick auf die Prognosen zum Fachkräftemangel zeigt, dass Einrichtungen heute noch keine, morgen aber schon massive Probleme haben können. Es gilt heute zu agieren, statt später nur noch reagieren zu können!

Employer Branding bietet ein schlüssiges Konzept, das in der freien Wirtschaft seine Funktionalität unter Beweis gestellt hat und eine langfristige Lösung gegen die Diagnose »Fachkräftemangel « für soziale Einrichtungen darstellt. 